\title{
Editorial
}

\section{La nueva configuración de las especialidades médicas en relación con la medicina del trabajo}

Sin duda alguna, uno de los mayores cambios que se ha producido en los últimos años en la formación de especialistas en ciencias de la salud, ha sido el que se refiere a la formación de los especialistas en Medicina del Trabajo.

Esta transformación, propiciada por la Ley de Prevención de Riesgos Laborales, supone una potenciación de las funciones y responsabilidades de los profesionales que ejercen en el ámbito de la salud laboral y consecuentemente llevó asociada importantes cambios en el proceso de formación de los especialistas en Medicina del Trabajo.

Se pasó del sistema de formación en régimen de "alumnado" al de "residencia", lo que supuso también una transformación esencial en la configuración de las nuevas unidades docentes, al integrar un conjunto de dispositivos educativos (escuelas profesionales, departamentos universitarios, Escuela Nacional de Medicina del Trabajo), asistenciales (hospitales y centros de atención primaria del Sistema Nacional de Salud), servicios de prevención y otras unidades colaboradoras pertenecientes a la administración autonómica o a la administración general del Estado.

La publicación, en mayo de 2005, de un nuevo y ambicioso programa formativo, orientado a la adquisición de conocimientos, competencias y habilidades, el incremento de la duración de la especialización, de tres a cuatro años y el incremento de las unidades docentes acreditadas y del número de plazas de formación ofertadas, constituyen hitos que reflejan la sensibilidad en materia de protección de la salud de los trabajadores y, por ende, el reconocimiento del papel de los médicos de la especialidad.

Este proceso de afianzamiento de la formación de los futuros médicos del Trabajo no ha concluido, pero se están dando pasos para lograrlo, como son los avances producidos en la reciente convocatoria de plazas de formación sanitaria especializada, cuyos residentes iniciarán su formación en el año 2009:

- En primer lugar se ha conseguido normalizar la oferta en medicina del trabajo, al publicarse junto con la del resto de las especialidades médicas, superándose así las dificultades existentes en años previos e integrándose esta especialidad totalmente en el sistema de residencia.

- Por otra parte se han convocado 135 plazas, lo cual supone seguir en la línea incremental de los últimos años (ver anexo 1).

- Además se ha aumentado tanto el número de unidades docentes, como los dispositivos asistenciales que integran cada una de ellas. De tal forma que, en este momento, prácticamente todas las comunidades autónomas cuentan con una unidad docente acreditada. 
Evidentemente debemos seguir trabajando para aumentar tanto el número de plazas como la calidad del sistema formativo, lo cual se conseguirá con el esfuerzo conjunto y coordinado de todas las administraciones implicadas, Ministerio de Sanidad y Consumo, Ministerio de Trabajo e Inmigración y Comunidades Autónomas.

Este proceso además se completará con el desarrollo de la especialidad de Enfermería del Trabajo y la formación de estos futuros profesionales en las mismas unidades docentes que los médicos, a través de la figura de las unidades docentes multiprofesionales que se estableció en el Real Decreto 183/2008, de 8 de febrero, por el que se determinan y clasifican las especialidades en Ciencias de la Salud y se desarrollan determinados aspectos del sistema de formación sanitaria especializada.

La Comisión Nacional de esta especialidad tiene ya elaborada una propuesta de programa formativo que en los próximos meses se someterá a revisión, de tal forma que una vez que este aprobado dicho programa podamos comenzar a definir los criterios de acreditación de las unidades docentes y avanzar así en la formación de estos futuros especialistas de enfermería, que tan necesarios son en el mercado laboral.

Pero además todos estos cambios en el ámbito de las especialidades sanitarias relacionadas con la salud laboral, tienen que vincularse e integrarse en la modificación sustancial del sistema de formación sanitaria especializada, en la que está trabajando el Ministerio de Sanidad y Consumo junto con las Comunidades Autónomas.

El sistema de residencia, que tan estupendos profesionales ha formado a los largo de más de 25 años, debe adaptarse a la definitiva consolidación del Estado de las autonomías y modernizarse, con el fin de conseguir una formación especializada más flexible y permeable que favorezca, al mismo tiempo, una visión multiprofesional y multidisciplinar de conjunto, más acorde con la realidad de nuestros días, sin perder por ello los grandes logros conseguidos que han hecho que la formación de especialistas haya sido una de las claves del reconocido prestigio y alto nivel profesional y científico que actualmente tiene nuestro Sistema Nacional de Salud.

Por esta razón, además del desarrollo del anteriormente citado RD 183/2008, que ha introducido criterios de racionalización y cambios sustanciales en el sistema de aprendizaje, de tutorización y de evaluación de los residentes, se ha comenzado ya a trabajar en el diseño de un modelo troncal de formación especializada, en desarrollo del artículo 19 de la Ley de Ordenación de las Profesiones Sanitarias.

El desarrollo de la troncalidad va a suponer un cambio cualitativo trascendente ya que al tiempo que se amplía y diversifica el contenido de la formación sanitaria especializada, tendremos un sistema de especialidades más flexible, capaz de dar respuesta a las futuras necesidades sociales y sanitarias y de favorecer las propias necesidades individuales de formación o de reorientación profesional.

Este cambio abrirá la posibilidad de inclusión de la Medicina del Trabajo en un tronco formativo común a especialidades afines, con contenidos eminentemente clínicos y sociales y aunque inevitablemente conllevará una modificación del programa de la especialidad, sin duda mejorará la formación e integración de estos profesionales en el sistema sanitario. 
Por otra parte la necesidad de dirigir la formación médica actual hacia la adquisición de competencias y el cambio demográfico de los aspirantes que se presentan a la prueba de acceso a la formación sanitaria especializada, ha determinado la necesidad de reformar la mencionada prueba. Esta reforma, que se ha iniciado en la presente convocatoria con la inclusión de la denominada "renuncia previa”, necesariamente nos debe llevar a completar la prueba actual e integrar en la misma la evaluación de las habilidades clínicas y comunicativas de los aspirantes.

La mejora del proceso de formación especializada de los profesionales debe ser una de las prioridades de cualquier sistema sanitario, porque sólo a través de la excelencia en la formación conseguiremos mejorar la calidad del proceso asistencial y permitir la mejor adecuación de los conocimientos profesionales a la evolución científica y técnica y a las necesidades sanitarias de la población.

El pasado 19 de septiembre se publicó la Orden de convocatoria de plazas de formación sanitaria especializada que dará entrada, en los próximos meses, a la cuarta promoción de residentes de Medicina del Trabajo. Sin duda alguna podemos congratularnos de la consolidación paulatina de un sistema de formación que utiliza los mejores recursos disponibles -humanos y materiales- y que está destinado a proveer al sistema sanitario de los profesionales más prestigiosos y mejor formados.

\begin{tabular}{|l|l|c|}
\hline \multicolumn{1}{|c|}{ COMUNIDAD } & \multicolumn{1}{c|}{ UNIDAD DOCENTE } & $\begin{array}{c}\text { PLAZAS } \\
\text { OFERTADAS }\end{array}$ \\
\hline ANDALUCÍA & U.D. M.T. ANDALUCÍA & 21 \\
\hline ARAGÓN & U.D. M.T. ARAGÓN & 7 \\
\hline CANTABRIA & U.D. M.T. CANTABRIA & 3 \\
\hline CASTILLA-LA MANCHA & U.D. M.T. CASTILLA-LA MANCHA & 6 \\
\hline CATALUÑA & U.D. M.T. UNIVERSIDAD DE BARCELONA & 8 \\
\hline CATALUÑA & U.D. M.T. MATEU ORFILA & 15 \\
\hline GALICIA & U.D. M.T. GALICIA & 3 \\
\hline MURCIA & U.D. M.T. MURCIA & 3 \\
\hline LA RIOJA & U.D. M.T. LA RIOJA & 2 \\
\hline ILLES BALEARS & U.D. M.T. ILLES BALEARS & 4 \\
\hline EUSKADI & U.D. M.T. EUSKADI & 5 \\
\hline ASTURIAS & U.D. M.T. PRINCIPADO DE ASTURIAS & 4 \\
\hline CASTILLA Y LEÓN & U.D. M.T. CASTILLA Y LEÓN & 30 \\
\hline MADRID & U.D. M.T. MADRID-1 & 12 \\
\hline MADRID & U.D. M.T. MADRID-2 & $\mathbf{1 3 5}$ \\
\hline NAVARRA & U.D. M.T. NAVARRA & \\
\hline VALECIA & U.D. M.T. VALENCIA & TOTAL \\
\hline & & \\
\hline
\end{tabular}

Para información más detallada de los dispositivos asistenciales que integran cada unidad docente ver BOE de 19 de septiembre de 2008, suplemento, páginas 168-177. 